\title{
Disparities in survival following surgery among patients with different histological types of N2-III non-small cell lung cancer: a Surveillance, Epidemiology and End Results (SEER) database analysis
}

\author{
Gang Jin ${ }^{1,2}$, Xin Wang ${ }^{3}$, Cai Xu ${ }^{1}$, Jifeng Sun ${ }^{1}$, Zhiyong Yuan ${ }^{1}$, Jun Wang ${ }^{1}$, Lujun Zhao ${ }^{1}$ \\ ${ }^{1}$ Department of Radiation Oncology, Tianjin Medical University Cancer Institute and Hospital, Tianjin, China; ${ }^{2}$ Department of Medical Oncology, \\ Second Hospital of Shanxi Medical University, Taiyuan, China; ${ }^{3}$ Department of Epidemiology and Biostatistics, First Affiliated Hospital, Army \\ Medical University, Chongqing, China \\ Contributions: (I) Conception and design: L Zhao, J Wang; (II) Administrative support: Z Yuan; (III) Provision of study materials or patients: G Jin; (IV) \\ Collection and assembly of data: J Sun, C Xu; (V) Data analysis and interpretation: X Wang, G Jin; (VI) Manuscript writing: All authors; (VII) Final \\ approval of manuscript: All authors. \\ Correspondence to: Jun Wang, MD; Lujun Zhao, MD. Department of Radiation Oncology, Tianjin Medical University Cancer Institute and Hospital. \\ No. 1 Tiyuanbei Road, Hexi District, Tianjin 300060, China. Email: WJUN800@126.com; zhaolujun@tjmuch.com.
}

\begin{abstract}
Background: This study aimed to determine the extent to which the survival outcomes of patients with N2-III non-small cell lung cancer (NSCLC) following surgery differ by histological subtype.

Methods: Patients with N2-III NSCLC receiving surgery between 2010 to 2016 were included using the Surveillance, Epidemiology and End Results (SEER) database. Cox proportional hazards models were used to identify risk factors associated with overall survival (OS) and non-cancer mortality. The KaplanMeier method with log-rank tests was used to estimate survival. Propensity score matching (PSM) was used. Statistical significance was defined as $\mathrm{P}<0.05$. Statistical analyses were done with IBM SPSS 23.0.

Results: Ultimately, 2,501 patients with stage N2-III NSCLC receiving surgery were included: 1,891 (75.6\%) patients had adenocarcinoma (AC), and 610 (24.4\%) patients had squamous cell cancer (SCC). The percentages of patients with AC and SCC receiving chemotherapy and postoperative radiotherapy (PORT) were comparable. In multivariate analysis, histology remained a significant predictor for OS and non-cancer mortality after adjusting for other clinical factors $(\mathrm{P}<0.05)$. Based on clinical factors, 522 patients with SCC were ultimately matched with 518 patients with AC using PSM. The 5-year OS of SCC patients after matching was much worse than that of $\mathrm{AC}$ patients $(36.3 \%$ vs. $41.5 \% ; \mathrm{P}=0.018)$, and the 5 -year non-cancer mortality of SCC patients was much higher than that of AC patients (18.8\% vs. $4.8 \%$; $\mathrm{P}=0.001)$.

Conclusions: Among patients with stage N2-III NSCLC following surgery, those with SCC had worse OS than those with AC, due to the higher percentage of patients dying from non-cancer causes.
\end{abstract}

Keywords: Squamous cell cancer (SCC); adenocarcinoma (AC); overall survival (OS); non-cancer mortality

Submitted May 12, 2020. Accepted for publication Aug 18, 2020.

doi: 10.21037/atm-20-4357

View this article at: http://dx.doi.org/10.21037/atm-20-4357

\section{Introduction}

For patients with operable stage N2-III non-small cell lung cancer (NSCLC), surgery remains the standard treatment. Furthermore, for these patients, adjuvant chemotherapy is routinely recommended, whereas, postoperative radiotherapy (PORT) is not routinely recommended owing to the lack of high level evidence, although it is still often used in clinical practice $(1,2)$. Despite the treatments, the overall survival (OS) of these patients continues to be poor, 
with a 5 -year OS of less than $25 \%$ (3). NSCLC comprises a group of malignancies, such as adenocarcinoma (AC), squamous cell cancer (SCC), neuroendocrine cancer, and large cell cancer, and whether histology should play a role in therapeutic decision making remains controversial. AC and SCC, which have long been considered to be almost similar in both postoperative prognosis and chemoradiotherapeutic response, account for approximately $85 \%$ of all NSCLC cases $(4,5)$.

Given the improvement of survival outcomes afforded by chemotherapy and the significant toxicity associated with chemotherapy and PORT, a better evaluation which incorporates the histological subtype of NSCLC may improve clinical decision making (6-8). In addition, chemotherapy responses vary according to different chemotherapy agents for histological subtypes of NSCLC. For instance, it was reported that a chemotherapeutic regimen of cisplatin and gemcitabine was more effective for SCC, whereas, a regimen of cisplatin and pemetrexed was more effective for AC (9). At present, the new agents of target therapy and immune therapy have significantly improved survival and safety profiles of patients with NSCLC (10) and increased the possibility for patients with stage N2-III to receive more aggressive and individualized treatment.

Currently, clinical decisions for NSCLC patients are still based on tumor-node-metastasis (TNM) stage, with no consideration given to histological subtype. Even though Grosu et al. reported that among patients with stage I NSCLC, those with SCC had a higher risk of mortality than those with $\mathrm{AC}$ taking into account competing risks (11). Whereas, the treatment modalities of patients with stage I NSCLC differ significantly from those with N2-III NSCLC. A randomized phase II trial by Yue $e t$ al. reported that adjuvant erlotinib improved 2-year disease-free survival in patients with EGFR mutation-positive stage IIIA NSCLC, compared with chemotherapy (81.4\% vs. 44.6\%; $\mathrm{P}=0.005)(10)$. Thus, whether histological subtype affects survival for patients with stage N2-III NSCLC receiving surgery remains unclear. While the recurrence rate is comparable between patients with AC and SCC following surgery (12). Our previous study showed that the recurrence pattern was different between patients with N2-IIIA AC and those with SCC, and patients with SCC tend to have mediastinal lymph nodes recurrence, whereas, those with AC tend to have distant metastasis (13). Several studies have reported that patients receiving radical resection with SCC had a poorer OS compared to those with AC $(12,14,15)$.
However, whether histological subtype affects survival for patients with stage N2-III NSCLC who receive surgery remains unclear.

The main objective of this study was to determine the extent to which the survival outcomes of patients with stage N2-III NSCLC following surgery differ by histological subtype. To this end, we compared the survival outcomes of patients with AC vs. SCC using the data from Surveillance, Epidemiology and End Results (SEER) database. We present the following article in accordance with the STROBE reporting checklist (available at http://dx.doi. org/10.21037/atm-20-4357).

\section{Methods}

\section{Data source}

The data for this population-based, retrospective study was abstracted from the SEER 18 registries research database, comprising information on approximately $30 \%$ of the total U.S. population. In the past decade, great advances in tumor management have been achieved. To explore the management and survival outcomes during the modern era and unify the tumor staging, lung cancer patients who were diagnosed between 2010 and 2016 were included in the present study. SEER*Stat Software version 8.3.6 was used to generate the case listing.

\section{Cobort selection}

The inclusion criteria were as follows: (I) diagnosed as the first and only malignant cancer; (II) only one primary site; (III) diagnosis not obtained from a death certificate or an autopsy; (IV) aged $\geq 18$ years; (V) having either a lobectomy or pneumonectomy; (VI) American Joint Committee on Cancer (AJCC, 7th edition) stage N2-III; (VII) AC and SCC; (VIII) receiving postoperative external-beam irradiation or no irradiation; (IX) stated number of lymph nodes involved and sampled; $(\mathrm{X})$ stated T tumor stage and laterality; and (XI) applicable cause of death. The exclusion criteria were as follows: (I) stage N0, N1, N3 diseases or unknown nodal status; (II) metastatic disease or unknown $\mathrm{T}$ tumor stage; (III) use of radiotherapy as preoperative radiotherapy, intraoperative radiotherapy, or brachytherapy; (IV) having wedge resection or segmentectomy; (V) other histological types apart from AC and SCC (e.g., small cell cancer, neuroendocrine cancer); and (VI) no cause of death listed. The flowchart for the population selection is shown in Figure 1. 


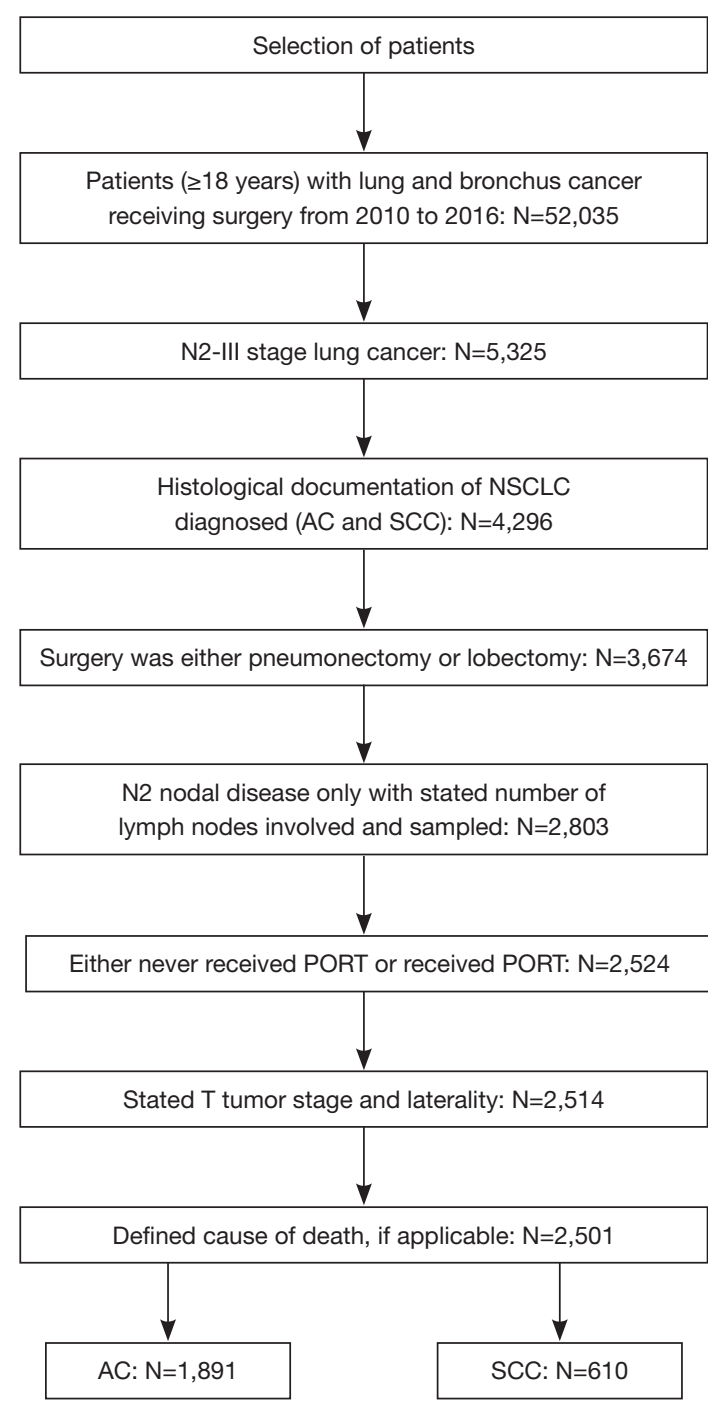

Figure 1 The flowchart for the population selection.

\section{Statistical analysis}

OS was defined as the time from the date of diagnosis to the date of death or last follow-up. For time to death from non-cancer, we used a Cox model to assess the causespecific hazard associated with the risk factors for death from non-cancer. The Kaplan-Meier method was used to estimate survival, with log-rank tests used to compare curves. Univariate analysis (UVA) and multivariate analysis (MVA) were done with Cox proportional hazards models. Covariates with $\mathrm{P}<0.2$ in the UVA were entered into the MVA models. A stepwise forward approach (likelihood ratio) was used to obtain the final MVA model. To reduce bias resulting from the retrospective nature of this study and to enhance comparability between groups, propensity score matching (PSM) analysis was used with Stata 14. Chisquare test was used to test the difference between groups. Statistical significance was defined as $\mathrm{P}<0.05$. Statistical analyses were completed with IBM SPSS 23.0 (IBM, Chicago, IL, USA).

\section{Results}

\section{Patient characteristics}

In all, 2,501 patients with stage N2-III NSCLC receiving surgery were included in the final analysis: 1,891 (75.6\%) patients had AC, and 610 (24.4\%) patients had SCC. The patient characteristics for the entire cohort are shown in Table 1 . The median age was 66 years (range, $18-89$ years), the majority were White $(80.3 \%)$, and most patients had early $\mathrm{T}$ tumor stage (75.6\%). Over a half of the patients had adequately sampled lymph nodes, and $41 \%$ patients had no fewer than 4 positive lymph nodes.

\section{The outcomes of univariate and multivariate analysis for overall survival}

The median survival time for all patients was 41 months (range, $0-83$ months), and the 5 -year OS was $38.9 \%$. The results of the UVA and MVA of factors for OS are summarized in Table 2. Under UVA, younger age, female, AC, early $\mathrm{T}$ stage, fewer positive lymph nodes [1-3], chemotherapy, PORT, and lobectomy were significantly associated with better $\mathrm{OS}(\mathrm{P}<0.05)$. The factors of interest that were identified from UVA with a $\mathrm{P}$ value $<0.2$ were entered into the MVA models. In the multivariate models for OS, younger age $(\mathrm{P}<0.001)$, female sex $(\mathrm{P}<0.001)$, AC $(\mathrm{P}=0.004)$, early $\mathrm{T}$ stage $(\mathrm{P}<0.001)$, localization in the left lung $(\mathrm{P}=0.019)$, fewer positive lymph nodes $[1-3](\mathrm{P}<0.001)$, chemotherapy $(\mathrm{P}<0.001)$, and lobectomy $(\mathrm{P}=0.018)$ were significantly associated better OS. However, PORT was not associated with OS $(\mathrm{P}=0.408)$.

\section{The outcomes of univariate and multivariate analysis for non-cancer mortality}

The results of the UVA and MVA for factors of non-cancer mortality are also summarized in Table 2. Under UVA, younger age, female sex, non-White and non-Black race, AC, chemotherapy, and PORT were significantly associated with lower non-cancer mortality $(\mathrm{P}<0.05)$. In multivariate 
Table 1 The patient characteristics of the entire cohort

\begin{tabular}{|c|c|}
\hline Variables & No. of patients [\%] \\
\hline \multicolumn{2}{|l|}{ Age (years) } \\
\hline$\leq 60$ & $742[30]$ \\
\hline 60 to 70 & 899 [36] \\
\hline$>70$ & $860[34]$ \\
\hline \multicolumn{2}{|l|}{ Sex } \\
\hline Male & $1,214[49]$ \\
\hline Female & $1,287[51]$ \\
\hline \multicolumn{2}{|l|}{ Race } \\
\hline White & $2,008[80]$ \\
\hline Black & $244[10]$ \\
\hline Other/unknown & 249 [10] \\
\hline \multicolumn{2}{|l|}{ Histology } \\
\hline Adenocarcinoma & $1,891[76]$ \\
\hline Squamous cell cancer & $610[24]$ \\
\hline \multicolumn{2}{|l|}{ T stage } \\
\hline $\mathrm{T} 1-2$ & $1,902[76]$ \\
\hline T3-4 & 599 [24] \\
\hline \multicolumn{2}{|l|}{ Laterality } \\
\hline Right & $1,396[56]$ \\
\hline Left & $1,105[44]$ \\
\hline \multicolumn{2}{|l|}{ No. of nodes sampled } \\
\hline$>10$ & $1,428[57]$ \\
\hline$\leq 10$ & $1,073[43]$ \\
\hline \multicolumn{2}{|c|}{ No. of positive lymph nodes } \\
\hline 1 to 3 & $1,483[59]$ \\
\hline$\geq 4$ & $1,018[41]$ \\
\hline \multicolumn{2}{|l|}{ Chemotherapy } \\
\hline Yes & $1,923[77]$ \\
\hline No & 578 [23] \\
\hline \multicolumn{2}{|l|}{ Postoperative radiotherapy } \\
\hline Yes & $1,049[42]$ \\
\hline No & $1,452[58]$ \\
\hline \multicolumn{2}{|l|}{ Type of surgery } \\
\hline Lobectomy & $2,285[91]$ \\
\hline Pneumonectomy & $216[9]$ \\
\hline
\end{tabular}

models for non-cancer mortality, younger age $(\mathrm{P}=0.015)$, non-White and non-Black race $(\mathrm{P}=0.038)$, chemotherapy $(\mathrm{P}<0.001)$, and $\mathrm{AC}(\mathrm{P}<0.001)$ were significantly associated with lower non-cancer mortality. However, sex $(\mathrm{P}=0.057)$ and PORT $(\mathrm{P}=0.958)$ did not impact non-cancer mortality.

\section{Propensity score matching for patients with squamous cell cancer and adenocarcinoma}

The number of patients with AC was twice that of the patients with SCC. Compared with SCC, AC had more young patients, more female patients, fewer White patients, more early $\mathrm{T}$ stage patients, and more patients with $\geq 4$ positive lymph nodes. Additionally, SCC had more patients receiving pneumonectomy than $\mathrm{AC}$, possibly due to the higher frequency of patients with centrally localized SCC. The percentage of $\mathrm{AC}$ patients receiving chemotherapy and PORT was comparable to that of SCC patients.

To reduce the influence of potential confounders in the comparisons between SCC and AC, PSM based on sex, race, age, PORT, laterality, T stage, number of positive lymph nodes, chemotherapy, and surgery types was used to create well-balanced groups. We ultimately matched 522 patients with SCC with 518 patients with AC (1:1 ratio, caliper 0.001). Characteristics of all eligible cases and PSM pairs are summarized in Table 3. The 5-year OS of patients with SCC after matching was much worse than that of AC patients (36.3\% vs. $41.5 \%$; $\mathrm{P}=0.018)$, and the 5 -year noncancer mortality of SCC patients with was much higher than that of AC patients ( $18.8 \%$ vs. $4.8 \%$; $\mathrm{P}=0.001)$. The Kaplan-Meier curves for OS by histological types are displayed in Figure 2.

The study was conducted in accordance with the Declaration of Helsinki (as revised in 2013).

\section{Discussion}

Our findings suggest that the histological subtype of stage N2-III NSCLC was an important prognostic indicator for OS. Patients with SCC had a much higher non-cancer mortality than those with AC, after adjusting for other variables.

For patients with N2-III NSCLC following surgery, the percentage of SCC patients who received chemotherapy and PORT was comparable to that of AC patients. This can be explained by the fact that NSCLC is always treated as a group of malignancies, without accounting for histology. 
Table 2 The results of univariate and multivariate analysis of factors for overall survival and non-cancer mortality

\begin{tabular}{|c|c|c|c|c|c|c|c|c|}
\hline \multirow{3}{*}{ Variables } & \multicolumn{4}{|c|}{ Univariate analysis } & \multicolumn{4}{|c|}{ Multivariate analysis } \\
\hline & \multicolumn{2}{|c|}{ OS } & \multicolumn{2}{|c|}{ Non-cancer mortality } & \multicolumn{2}{|c|}{ OS } & \multicolumn{2}{|c|}{ Non-cancer mortality } \\
\hline & $\mathrm{HR}$ & $\mathrm{P}$ & $\mathrm{HR}$ & $\mathrm{P}$ & $\mathrm{HR}$ & $\mathrm{P}$ & $\mathrm{HR}$ & $\mathrm{P}$ \\
\hline Age (years) & 1.365 & $<0.001$ & 1.647 & $<0.001$ & 1.299 & $<0.001$ & 1.354 & 0.015 \\
\hline \multicolumn{9}{|l|}{$\leq 60$} \\
\hline \multicolumn{9}{|l|}{60 to 70} \\
\hline \multicolumn{9}{|l|}{$>70$} \\
\hline Sex & 1.517 & $<0.001$ & 1.501 & 0.025 & 1.466 & $<0.001$ & & 0.057 \\
\hline \multicolumn{9}{|l|}{ Male } \\
\hline \multicolumn{9}{|l|}{ Female } \\
\hline \multicolumn{9}{|l|}{ Other/unknown } \\
\hline Histology & 1.337 & $<0.001$ & 2.117 & $<0.001$ & 1.225 & 0.004 & 2.135 & $<0.001$ \\
\hline \multicolumn{9}{|l|}{$A C$} \\
\hline \multicolumn{9}{|l|}{ SCC } \\
\hline T stage & 1.408 & $<0.001$ & & 0.33 & 1.32 & $<0.001$ & & \\
\hline \multicolumn{9}{|l|}{$\mathrm{T} 1-2$} \\
\hline \multicolumn{9}{|l|}{ T3-4 } \\
\hline \multicolumn{9}{|l|}{$\leq 10$} \\
\hline No. of positive lymph nodes & 1.375 & $<0.001$ & & 0.375 & 1.418 & $<0.001$ & & \\
\hline \multicolumn{9}{|l|}{1 to 3} \\
\hline \multicolumn{9}{|l|}{$\geq 4$} \\
\hline Chemotherapy & 0.549 & $<0.001$ & 0.274 & $<0.001$ & 0.576 & $<0.001$ & 0.3 & $<0.001$ \\
\hline \multicolumn{9}{|l|}{ Yes } \\
\hline No & & & & & & & & \\
\hline PORT & 0.795 & $<0.001$ & 0.615 & 0.011 & & 0.408 & & 0.958 \\
\hline Yes & & & & & & & & \\
\hline No & & & & & & & & \\
\hline Type of surgery & 1.466 & $<0.001$ & & 0.439 & 1.263 & 0.018 & & \\
\hline Lobectomy & & & & & & & & \\
\hline Pneumonectomy & & & & & & & & \\
\hline
\end{tabular}

PORT, postoperative radiotherapy; OS, overall survival; AC, adenocarcinoma; SCC, squamous cell cancer. 
Table 3 Characteristics of all eligible cases and propensity score matching pairs for patients with adenocarcinoma and squamous cell cancer

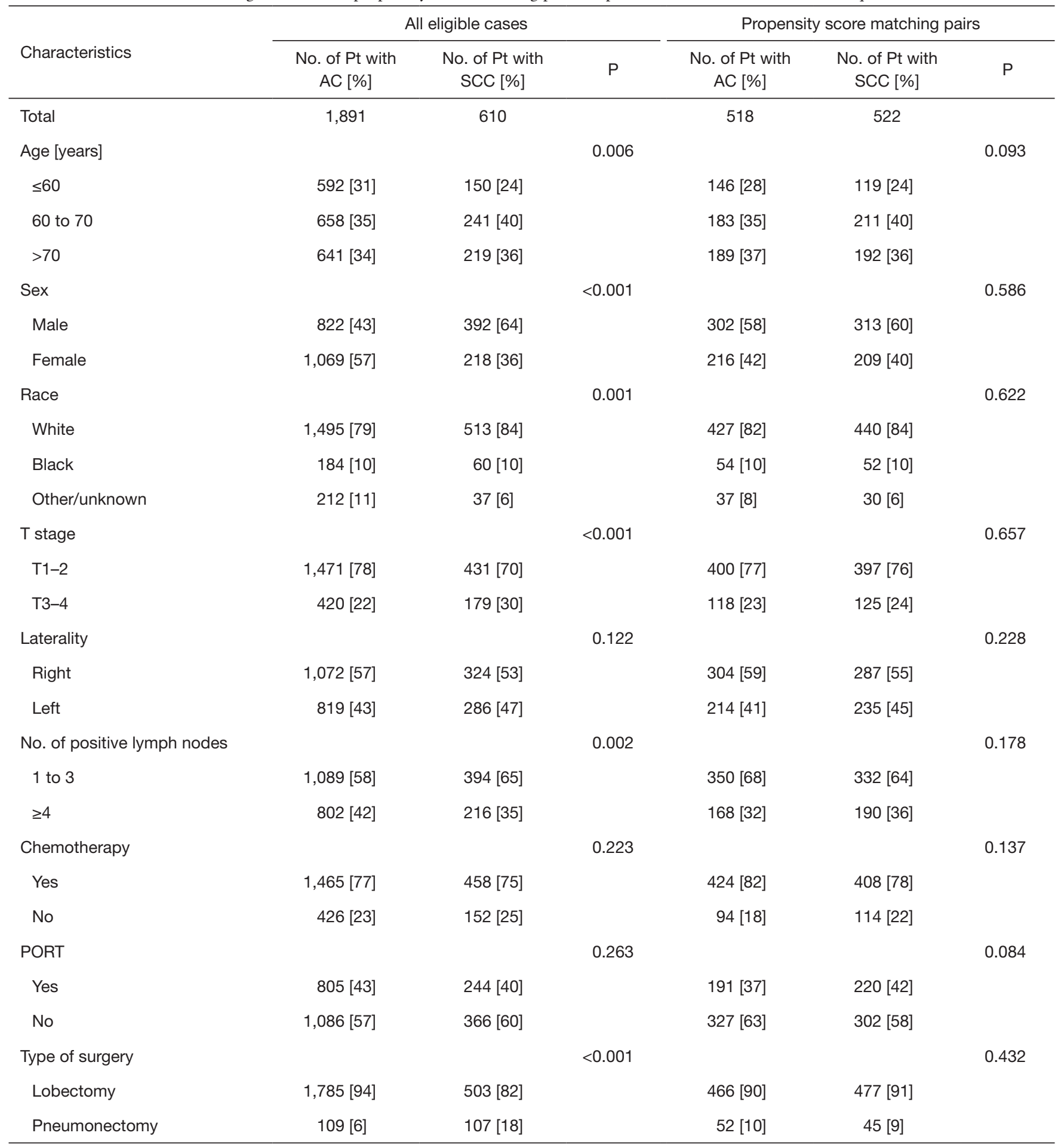

PORT, postoperative radiotherapy; Pt, patients; AC, adenocarcinoma; SCC, squamous cell cancer. 


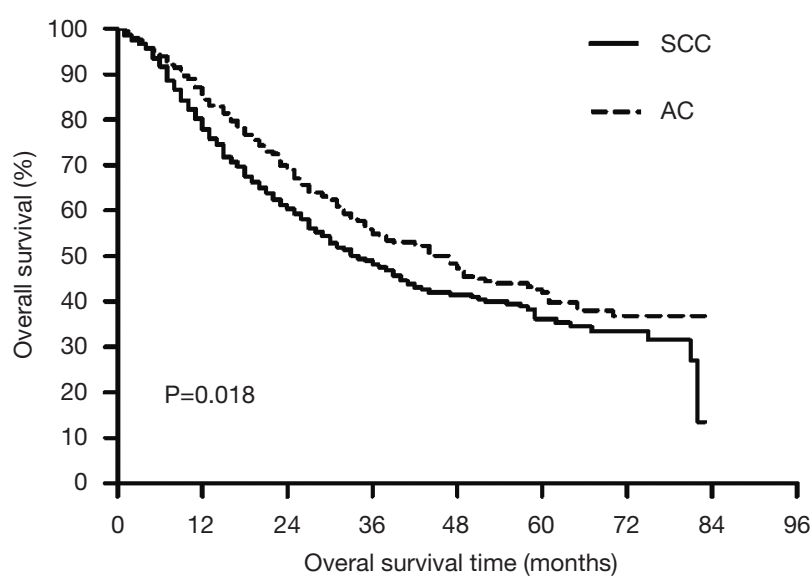

Figure 2 Overall survival of patients with adenocarcinoma (AC) and squamous cell cancer (SCC) who had N2-III stage non-small cell lung cancer receiving surgery.

In addition, the target therapy is not recorded in the SEER database, although a great number of patients with $\mathrm{AC}$ who have mutation-driven genes receive target therapy (10). Furthermore, more SCC patients receive pneumonectomy than AC patients do, which may be a result of the higher proportion of patients with SCC located centrally in the mediastinum.

The survival rate results for AC and SCC in patients undergoing complete surgery are mixed. Some research suggests that SCC histology has worse survival than AC histology. In the study by Nakamura et al., 537 NSCLC patients with surgery (AC, 434; SCC, 103) were included, with SCC having a poorer OS compared to the AC histology (12). A study using the SEER database reported that histology was a significant predictor for survival (16). In contrast, several studies have demonstrated that NSCLC patients with SCC had better survival than those with AC $(17,18)$. The Adjuvant Navelbine International Trialist Association (ANITA) trial reported the survival of NSCLC patients with completely resected stage IB-IIIA diseases who were randomly assigned to vinorelbine and cisplatin or to observation (17). The trial yielded a median survival time of 37.3 and 45.5 months for patients with AC and non$\mathrm{AC}$, respectively, and AC histology appeared to be a poor prognostic factor in patients with resected NSCLC (18). After analyzing 9,137 surgically managed cases with NSCLC, Chansky et al. found a small survival advantage for SCC over AC (19). In contrast, the study by Douillard, etc. showed no survival difference between patients with SCC and non-SCC (8).
In this study, after adjusting for other clinical variables, including, sex, race, age, PORT, laterality, T stage, number of positive lymph nodes, chemotherapy, and surgery types, we found that SCC histology had worse OS than AC histology for patients with N2-III NSCLC receiving surgery, which is similar to the results of previously published studies, to some extent $(12,14,15,20)$. After PSM, the 5-year OS for patients with SCC was 5.2\% lower than that of $\mathrm{AC}(\mathrm{P}=0.018)$. This may be attributable to SCC tending to have a greater loco-regional failure rate than AC (HR 1.934, 95\% CI: 0.94-3.977, P=0.073) (21), which contributes to worse OS. Unfortunately, recurrence information is not available on the SEER database, so we are not able to compare loco-regional failure between SCC and AC.

To further determine the reasons for the survival differences between SCC and AC, we analyzed the noncancer mortality as the complementary endpoint for OS. Surprisingly, we found that the non-cancer mortality of SCC was $14 \%$ higher than AC $(P=0.001)$. One of the reasons postulated to account for this survival difference is the fact that SCC had higher non-cancer mortality, which contributes to decreased survival. Another reason is the fact that patients with SCC are more likely to be smokers $(12,20)$. Smoking is a risk factor for recurrence (12), which contributes to decreased OS, and is associated with several life-threatening diseases, like emphysema, pneumonia, ischemic heart diseases, cerebrovascular disease, and several malignant tumors including lung cancer, all of which may result in a high risk of non-cancer mortality and decreased OS $(12,22,23)$.

This study is the first to compare the OS and non-cancer mortality of patients with SCC and AC who had N2-III NSCLC following surgery. Our findings revealed that the reason for SCC having a worse OS potentially lies in the fact that more patients with SCC die from non-cancer causes, such as chronic obstructive pulmonary disease, ischemic heart diseases, and cerebrovascular disease, rather than from the tumor itself. To reduce bias in this study, we included patients defined according to the AJCC 7th version guidelin es applied after 2010 to ensure that most patients were treated with relatively consistent and modern modalities.

Our study had several limitations, chief among them was its retrospective nature. Second, the SEER database did not have information on smoking, smoking-related comorbidities and death, other detailed causes of death, and tumor recurrence and related treatment, which limited 
our comparison of smoking-caused death and recurrencerelated death between patients with SCC and AC. Third, the SEER database did not have information concerning adjuvant therapy, including data on radiation dose, chemotherapy cycles, chemotherapy agents, etc. Fourth, the SEER database did not have information on target therapy, which limited our analysis on the benefits of target therapy for patients with AC. Given these restrictions, further prospective studies are needed in the future.

In conclusion, our study showed that among patients with stage N2-III NSCLC following surgery, the management modalities between SCC and AC histology were similar. Also, those with SCC histology had worse OS than those with AC as result of the higher percentage of patients dying from non-cancer causes. Thus, more aggressive and individualized treatment modalities should be taken to improve the therapeutic efficiency of patients with N2-III SCC.

\section{Acknowledgments}

Funding: None.

\section{Footnote}

Reporting Checklist: The authors have completed the STROBE reporting checklist. Available at http://dx.doi. org/10.21037/atm-20-4357

Conflicts of Interest: All authors have completed the ICMJE uniform disclosure form (available at http://dx.doi. org/10.21037/atm-20-4357). The authors have no conflicts of interest to declare.

Ethical Statement: The authors are accountable for all aspects of the work in ensuring that questions related to the accuracy or integrity of any part of the work are appropriately investigated and resolved. The study was conducted in accordance with the Declaration of Helsinki (as revised in 2013).

Open Access Statement: This is an Open Access article distributed in accordance with the Creative Commons Attribution-NonCommercial-NoDerivs 4.0 International License (CC BY-NC-ND 4.0), which permits the noncommercial replication and distribution of the article with the strict proviso that no changes or edits are made and the original work is properly cited (including links to both the formal publication through the relevant DOI and the license). See: https://creativecommons.org/licenses/by-nc-nd/4.0/.

\section{References}

1. NSCLC Meta-analyses Collaborative Group, Arriagada R, Auperin A, et al. Adjuvant chemotherapy, with or without postoperative radiotherapy, in operable non-small-cell lung cancer: two meta-analyses of individual patient data. Lancet 2010;375:1267-77.

2. Kris MG, Gaspar LE, Chaft JE, et al. Adjuvant Systemic Therapy and Adjuvant Radiation Therapy for Stage I to IIIA Completely Resected Non-Small-Cell Lung Cancers: American Society of Clinical Oncology/Cancer Care Ontario Clinical Practice Guideline Update. J Clin Oncol 2017;35:2960-74.

3. Van Schil PE, Rami-Porta R, Asamura H. The 8(th) TNM edition for lung cancer: a critical analysis. Ann Transl Med 2018;6:87.

4. Pisters KM, Evans WK, Azzoli CG, et al. Cancer Care Ontario and American Society of Clinical Oncology adjuvant chemotherapy and adjuvant radiation therapy for stages I-IIIA resectable non small-cell lung cancer guideline. J Clin Oncol 2007;25:5506-18.

5. Feng W, Fu XL, Cai XW, et al. Patterns of local-regional failure in completely resected stage IIIA(N2) non-small cell lung cancer cases: implications for postoperative radiation therapy clinical target volume design. Int J Radiat Oncol Biol Phys 2014;88:1100-7.

6. Burdett S, Rydzewska L, Tierney J, et al. Postoperative radiotherapy for non-small cell lung cancer. Cochrane Database Syst Rev 2016;10:CD002142.

7. Louie AV, Bahig H, Moghanaki D. The current and future landscape of radiotherapy for lung cancer. Transl Lung Cancer Res 2019;8:S122-3.

8. Douillard JY, Rosell R, De Lena M, et al. Impact of postoperative radiation therapy on survival in patients with complete resection and stage I, II, or IIIA non-smallcell lung cancer treated with adjuvant chemotherapy: the adjuvant Navelbine International Trialist Association (ANITA) Randomized Trial. Int J Radiat Oncol Biol Phys 2008;72:695-701.

9. Scagliotti GV, Parikh P, von Pawel J, et al. Phase III study comparing cisplatin plus gemcitabine with cisplatin plus pemetrexed in chemotherapy-naive patients with advanced-stage non-small-cell lung cancer. J Clin Oncol 2008;26:3543-51.

10. Yue D, Xu S, Wang Q, et al. Erlotinib versus vinorelbine 
plus cisplatin as adjuvant therapy in Chinese patients with stage IIIA EGFR mutation-positive non-small-cell lung cancer (EVAN): a randomised, open-label, phase 2 trial. Lancet Respir Med 2018;6:863-73.

11. Grosu HB, Manzanera A, Shivakumar S, et al. Survival disparities following surgery among patients with different histological types of non-small cell lung cancer. Lung Cancer 2020;140:55-8.

12. Nakamura H, Sakai H, Kimura H, et al. Difference in Postsurgical Prognostic Factors between Lung Adenocarcinoma and Squamous Cell Carcinoma. Ann Thorac Cardiovasc Surg 2017;23:291-7.

13. Qin PY, Yuan ZY, Wang J, et al. The postoperative recurrence pattern of patients with IIIA (N2) stage non-small cell lung cancer. Chinese Lung Cancer 2009; 12:1095-100.

14. Asamura H, Goya T, Koshiishi Y, et al. A Japanese Lung Cancer Registry study: prognosis of 13,010 resected lung cancers. J Thorac Oncol 2008;3:46-52.

15. Sawabata N, Miyaoka E, Asamura H, et al. Japanese lung cancer registry study of 11,663 surgical cases in 2004: demographic and prognosis changes over decade. J Thorac Oncol 2011;6:1229-35.

16. Zhang J, Gold KA, Lin HY, et al. Relationship between tumor size and survival in non-small-cell lung cancer (NSCLC): an analysis of the surveillance, epidemiology, and end results (SEER) registry. J Thorac Oncol 2015;10:682-90.

17. Douillard JY, Rosell R, De Lena M, et al. Adjuvant vinorelbine plus cisplatin versus observation in patients with completely resected stage IB-IIIA non-small-cell lung cancer (Adjuvant Navelbine International Trialist

Cite this article as: Jin G, Wang X, Xu C, Sun J, Yuan Z, Wang J, Zhao L. Disparities in survival following surgery among patients with different histological types of N2-III nonsmall cell lung cancer: a Surveillance, Epidemiology and End Results (SEER) database analysis. Ann Transl Med 2020;8(20):1288. doi: $10.21037 /$ atm-20-4357
Association [ANITA]): a randomised controlled trial. Lancet Oncol 2006;7:719-27.

18. Bennouna J, Senellart H, Hiret S, et al. Impact of histology on survival of resected non-small cell lung cancer (NSCLC) receiving adjuvant chemotherapy: subgroup analysis of the adjuvant vinorelbine (NVB) cisplatin (CDDP) versus observation in the ANITA trial. Lung Cancer 2011;74:30-4.

19. Chansky K, Sculier JP, Crowley JJ, et al. The International Association for the Study of Lung Cancer Staging Project: prognostic factors and pathologic TNM stage in surgically managed non-small cell lung cancer. J Thorac Oncol 2009;4:792-801.

20. Wang BY, Huang JY, Chen HC, et al. The comparison between adenocarcinoma and squamous cell carcinoma in lung cancer patients. J Cancer Res Clin Oncol 2020;146:43-52.

21. Amini A, Lou F, Correa AM, et al. Predictors for locoregional recurrence for clinical stage III-N2 nonsmall cell lung cancer with nodal downstaging after induction chemotherapy and surgery. Ann Surg Oncol 2013;20:1934-40.

22. Doll R, Peto R, Wheatley K, et al. Mortality in relation to smoking: 40 years' observations on male British doctors. BMJ 1994;309:901-11.

23. Qiang G, Liang C, Xiao F, et al. Impact of chronic obstructive pulmonary disease on postoperative recurrence in patients with resected non-small-cell lung cancer. Int J Chron Obstruct Pulmon Dis 2016;11:43-9.

(English Language Editor: J. Gray) 